\title{
Quantitative and structural factors in the judgment of pattern complexity
}

\author{
SHIN'ICHI ICHIKAWA \\ Saitama University, Urawa, Japan
}

\begin{abstract}
The processes underlying judgment of pattern complexity were investigated using patterns containing eight dots in a $4 \times 4$ matrix. Thirty-two subjects rated the complexity of 140 patterns that were taken from patterns generated by 12 other subjects in a preliminary experiment. Factor analysis of mean complexity ratings and 12 physical variables, which objectively described configuration of dots, revealed a "quantitative factor" and a "structural factor." Mean complexity loaded on both factors. Multiple regression analysis showed that the mean complexity was well predicted by combination of a quantitative variable with a structural variable. Analysis of individual data also suggested that individual differences in complexity judgment could be explained by the weights on these two factors. In addition, a rating experiment was carried out in which 60 patterns were presented in limited stimulus durations (50 msec, $200 \mathrm{msec}, 1 \mathrm{sec}$, or $4 \mathrm{sec}$ ). The quantitative variables were highly correlated with the complexity ratings in all conditions, whereas the structural variables affected the ratings in long durations but not in short durations. These results support the view that visual complexity is judged through two processes: One is a fast process that evaluates quantitative aspects in a stimulus pattern, and the other is a slow process that detects the structure in the pattern.
\end{abstract}

In the field of "psychophysics of form" (Attneave \& Arnoult, 1956) or "pattern psychophysics" (Ichikawa \& Gyoba, 1984), many researchers have studied what physical variables are useful in estimating psychological variables for visual patterns. Some recent studies have succeeded in approximating complexity, goodness, or memorability by multiple regression analysis using physical variables that describe two-dimensional dot patterns or block patterns (e.g., Chipman, 1977; Ichikawa, 1981; Yodogawa, 1982). However, many methodological problems have remained unresolved in these studies.

First, sampling of patterns has been arbitrary, although the influence of a predictor variable (i.e., the variance explained by the variable) is largely affected by the procedure of sampling. Second, the interrelation among physical variables has not been made clear. Third, mean ratings have been analyzed in many studies, but there have been few studies concerning individual differences in ratings. Fourth, the information processes underlying the judgment of pattern complexity have not been discussed sufficiently.

The present study consists of three parts. Part 1 describes the "subject-generating method" used to obtain

\footnotetext{
This research was partly supported by a Grant-in-Aid for Scientific Researches, Ministry of Education, Science, and Culture, No. 58310014 (representative: Tadasu Oyama, University of Tokyo). I thank Susan F. Chipman, Eiji Yodogawa, Shin'ichi Mayekawa, and Toshiro Inui for helping me to compute the physical variables used in this study. Thanks are also due to Tadasu Oyama of the University of Tokyo for his suggestions and comments on an earlier draft of this paper.

Requests for reprints should be sent to Shin'ichi Ichikawa, Information Processing Center, Saitama University, Shimo-Ohkubo, Urawa, Saitama 338, Japan.
}

a set of stimulus dot patterns which were of wide range of complexity and free from the intention of researchers. Part 2 describes a free rating experiment using a sample from this set and presents an analysis of the data. Multiple regression analysis shows what physical variables are useful for the prediction of complexity ratings, and the interrelation among physical variables is discussed based on the results of factor analysis. Individual differences are also analyzed by means of factor analysis in which the subjects are regarded as variables. Part 3 describes the effects of physical variables upon complexity ratings under the conditions of various stimulus durations. Here a tentative model is proposed for information processing in the judgment of visual complexity. The results of these analyses strongly suggest the validity of a framework of quantitative and structural factors as originally proposed by Chipman (1977).

\section{PART 1: PRELIMINARY SAMPLING OF PATTERNS}

The method of sampling stimulus patterns is one of the important problems in pattern psychophysics, because the effect of each physical variable upon complexity or goodness largely depends on the sampling method used. Garner and Clement (1963), Imai, T. Ito, and S. Ito (1976), and Yodogawa (1982) showed that pattern complexity or goodness was well accounted for by the variables relating to the degree of symmetry, but it should be noted that the stimulus patterns used in these experiments were chosen by the researchers. Attneave (1957), on the other hand, showed that the number of turns in a random polygon could explain $79 \%$ of the variance of complexity rat- 
ings, whereas the symmetry could explain only $4 \%$ of the variance. Thus, the effect of the same factor, symmetry, is very large in some studies but very small in other studies. One important reason for this seems to be the difference in methods of making or sampling stimulus patterns.

If researchers select patterns at will, they may, consciously or unconsciously, make a sample of patterns whose complexity ratings are mostly explained by the factors that the researchers regard as important. Such samples are, of course, biased. On the other hand, so-called "random patterns"' present a different problem. Consider the distribution of complexity of dot patterns which have $\mathrm{d}$ dots in an $\mathrm{n} \times \mathrm{n}$ matrix. If the population is considered, complex patterns are many while simple patterns are few. When sampled randomly (using a computer or a table of random numbers), most patterns are too complex, with the result that important factors may be missed. For example, there are many symmetrical shapes in our daily life, so we regard symmetry as one of the most important factors affecting visual complexity. However, symmetrical patterns are rarely made by means of a random procedure. In other words, random patterns are already controlled for the factor of symmetry. Consequently, researchers using only random patterns may conclude that symmetry is unimportant to the estimation of complexity, as shown in the study of Attneave (1957). This conclusion is apparently derived from patterns of biased range of complexity.

The subject-generating method was used in the present study in order to make a set of patterns that were of wide range of complexity and free from the intentions or hypotheses of researchers. In this method, it is not researchers but naive subjects that make patterns.

\section{Method}

Subjects. The subjects were 12 students, graduate and undergraduate, and they performed five trials each.

Materials. As shown in Figure 1, seven $4 \times 4$ matrices were drawn on a vertical white board. The size of the whole matrix was $16 \times 16 \mathrm{~cm}$; each cell was $4 \times 4 \mathrm{~cm}$. The numbers 1 through 7 , representing the grades of pattern complexity, were written above the matrices. Eight black magnetic disks, $2 \mathrm{~cm}$ in diameter, were positioned beside each matrix.

Procedure. The subjects were asked to make a series of eightdot patterns corresponding to the seven grades of complexity ("complexity grades"). In a 4-min trial, subjects were permitted to go back and forth from one matrix to another. Figure 1 shows the example patterns generated by a subject in a trial. An experimenter recorded the seven patterns at intertrial intervals of $1 \mathrm{~min}$. The sub-

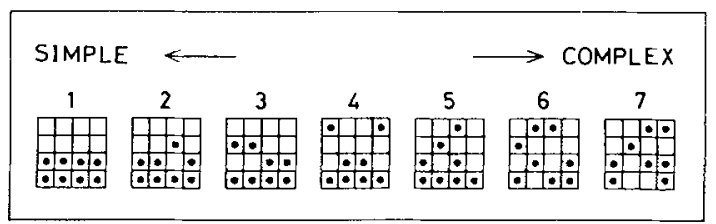

Figure 1. Patterns generated by Subject N.K. in Trial 1.
Table 1

Number of Patterns Generated by Subjects

\begin{tabular}{cc}
\hline Complexity Grade & Number \\
\hline $1.000-1.999$ & 20 \\
$2.000-2.999$ & 40 \\
$3.000-3.999$ & 50 \\
$4.000-4.999$ & 54 \\
$5.000-5.999$ & 55 \\
$6.000-6.999$ & 54 \\
7.000 & 50 \\
Total & 323 \\
\hline
\end{tabular}

jects were instructed to try not to generate a configuration that had been generated during previous trials. They could ask the experimenter whether a configuration was new or not.

\section{Results}

The subjects generated 420 patterns ( 7 patterns $\times$ 5 trials $\times 12$ subjects). However, the identical patterns were counted as one, and the complexity grade was defined as the mean of their complexity grades. As shown in Table 1, simple patterns were sometimes identical.

Thus we were able to make a set of patterns which were of wide range of complexity and free from the intentions or hypotheses of researchers. However, the complexity grade assigned here seemed to be too rough and unreliable as a measure of perceived complexity, since it was obtained through only one trial or was an average over only a few trials. It was necessary to get more data on complexity for each pattern. In Part 2, the method and the results of a rating experiment are described.

\section{PART 2: FREE RATING EXPERIMENT}

\section{Method}

Subjects. The subjects were 32 undergraduate students who had never participated in this kind of rating experiment.

Materials. From the 323 dot-in-matrix patterns generated, 140 patterns were randomly sampled with the restriction that 20 patterns should be selected from each of the seven complexity grades shown in Table 1. These patterns were printed on two sheets of paper. The size of each sheet was $25.6 \mathrm{~cm}$ (vertical) $\times 36.3 \mathrm{~cm}$ (horizontal). The 70 patterns on a sheet of paper were arranged 7 rows $\times 10$ columns in random order. The size of a matrix was $2 \times 2 \mathrm{~cm}$, and the diameter of a dot was $2.5 \mathrm{~mm}$. Parentheses were printed beside the patterns and subjects were asked to put their complexity ratings here.

Procedure. The subjects were asked to rate the complexity of the 140 patterns on a seven-point scale, with high scores representing complex patterns. Half of the subjects began with one sheet, and the other half with the other sheet. The subjects were given the task in groups. It took 15 to $25 \mathrm{~min}$ for a subject to complete the rating task.

\section{Analyses of Mean Complexity}

Means and standard deviations (SDs) of complexity ratings are presented in Table $2 .^{1}$ In this table, dot-inmatrix patterns are expressed by hexadecimal numbers. For example, the pattern of Grade 7 in Figure 1 is once encoded into a binary pattern " $0011,0100,1011,1001$ " by rows, starting at the top. Then it is encoded into a hexa- 
Table 2

Means and Standard Deviations of Complexity Ratings

\begin{tabular}{|c|c|c|c|c|c|c|c|c|c|c|c|}
\hline Pate & tern & Mean & SD & Patt & tern & Mean & SD & Patt & tern & Mean & $S D$ \\
\hline i* & OFFO & 1.031 & 0.174 & $51 *$ & $\operatorname{cc} 99$ & 3.094 & 0.914 & $101 *$ & DE 84 & 5.469 & 1.000 \\
\hline $2 *$ & FFOO & 1.031 & 0.174 & $52 \star$ & 3399 & 3.125 & 1.053 & 102 & $\mathrm{C} 3 \mathrm{D} 4$ & 5.469 & 1.145 \\
\hline 3 & 6666 & 1.031 & 0.174 & 53 & F898 & 3.188 & 0.950 & $103 *$ & $968 \mathrm{~B}$ & 5.531 & 1.250 \\
\hline 4 & 3333 & 1.031 & 0.174 & 54 & OC $3 F$ & 3.188 & 1.158 & 104 & $265 D$ & 5.594 & 0.996 \\
\hline 5 & OOFF & 1.063 & 0.242 & 55 & DD22 & 3.250 & 1.436 & $105 *$ & $5 A 59$ & 5.688 & 1.356 \\
\hline 6 & FOOF & 1.094 & 0.292 & 56 & $906 \mathrm{~F}$ & 3.250 & 1.250 & 106 & 9867 & 19 & 0.943 \\
\hline 7 & $\mathrm{CCCC}$ & 1.125 & 0.545 & 57 & EAA 8 & 3.281 & 1.007 & $107 \star$ & $5 \mathrm{C} / \mathrm{B}$ & & 1.253 \\
\hline 8 & 9999 & 1.250 & 0.612 & $58 *$ & $4 \mathrm{BFO}$ & 3.344 & 1.314 & 108 & D269 & 5.875 & 0.857 \\
\hline 9 & 5555 & 1.250 & 0.500 & 59 & $08 \mathrm{EF}$ & 3.375 & 1.111 & 109 & D4 39 & 5.906 & 0.980 \\
\hline $10 *$ & FOFO & 1.281 & 0.514 & 60 & $8 \mathrm{CCE}$ & 3.406 & 1.247 & $110 *$ & $695 \mathrm{~A}$ & 5.938 & 1.435 \\
\hline $11 *$ & $33 \mathrm{CC}$ & 1.563 & 0.899 & 61 & 08DF & 3.469 & 1.250 & 111 & E692 & 5.938 & 0.998 \\
\hline 12 & $\operatorname{cc} 33$ & 1.719 & 0.760 & $62 \star$ & $\mathrm{CBCD}$ & 3.500 & 1.369 & $112 \star$ & $395 \mathrm{~A}$ & 5.969 & 0.918 \\
\hline $13 *$ & 9669 & 2.094 & 1.042 & 63 & 9969 & 3.500 & 1.199 & $113 *$ & 5659 & 6.000 & 0.791 \\
\hline $14 \star$ & 9996 & 2.156 & 0.755 & $64 *$ & $2 \mathrm{E} 74$ & 3.563 & 1.456 & $114 *$ & $916 \mathrm{D}$ & 6. & 0.810 \\
\hline 15 & FE 10 & 2.188 & 0.682 & $65 *$ & $5 \mathrm{~A} 5 \mathrm{~A}$ & 3.594 & 1.869 & $115 *$ & BA54 & 6. & 0.810 \\
\hline 16 & 0757 & 2.219 & 0.9 & 66 & $398 \mathrm{E}$ & 3.781 & 1.3 & 116 & A96A & 6. & 1.144 \\
\hline 17 & $988 \mathrm{~F}$ & 2.219 & 0.992 & $67 *$ & 7358 & 3.906 & 1.665 & 117 & 2D78 & 6. & 0.966 \\
\hline $18 *$ & 6999 & 2.250 & 0.750 & 68 & SDDO & 4.031 & 1.132 & 118 & B436 & 6.063 & 0.998 \\
\hline 19 & 6996 & 2.250 & 1.000 & $69 *$ & D 18B & 4.094 & 1.528 & 1.19 & 9A5A & 6.125 & 1.139 \\
\hline $20 *$ & $99 \mathrm{FO}$ & 2.344 & 1.215 & 70 & $446 \mathrm{~F}$ & 4.125 & 1.083 & 120 & A5A9 & 6.156 & 1.034 \\
\hline 21 & 6665 & 2.344 & 0.690 & $71 *$ & A5A5 & 4.188 & 1.861 & $121 *$ & B36C & 6.188 & 0.808 \\
\hline 22 & $\operatorname{ccc} 9$ & 2.344 & 0.922 & $72 \star$ & EB88 & 4.219 & 0.992 & 122 & $4 A 5 B$ & 6.219 & 0.992 \\
\hline $23 *$ & $11 \mathrm{EE}$ & 2.375 & 0.893 & 73 & D22D & 4.250 & 1.173 & 123 & A693 & 6.219 & 1.082 \\
\hline 24 & F990 & 2.375 & 1.023 & 74 & CE62 & 4.281 & 1.125 & 124 & A569 & 6.250 & 1.031 \\
\hline 25 & $333 \mathrm{C}$ & 2.406 & 0.9 & $75 *$ & $8 \mathrm{CE} 3$ & 4.4 & 1. & 125 & $4 B 59$ & 3 & 26 \\
\hline 26 & EE I I & 2.406 & 0.9 & 76 & F05A & 4.406 & 1.2 & $126^{\star}$ & A56C & & \\
\hline $27 \star$ & 0776 & 2.438 & 0.788 & $77 \star$ & A9CC & 4.438 & 1.248 & 127 & D968 & 6.344 & 0.956 \\
\hline 28 & 9966 & 2.438 & 0.864 & $78 *$ & E2 IE & 4.469 & 1.145 & 128 & 58D6 & 6.375 & 0.992 \\
\hline 29 & F311 & 2.469 & 1.000 & 79 & $724 \mathrm{E}$ & 4.469 & 1.500 & 129 & A58D & 6.375 & 0.820 \\
\hline $30 \star$ & 9666 & 2.563 & 0.933 & 80 & $69 \mathrm{IE}$ & 4.500 & 1.299 & 130 & $5 A 96$ & 6.375 & 0.992 \\
\hline $31 *$ & $|E E|$ & 2.563 & 0.96 & 81 & O6CF & 4.531 & 1.323 & $|3| *$ & 4B5A & 6.438 & 0.747 \\
\hline $32 \star$ & OCCF & 2.563 & 0.78 & $82 *$ & FC24 & 4.563 & 0.998 & 132 & A35A & 6.469 & 0.790 \\
\hline 33 & 3339 & 2.563 & 1.2 & 83 & 6604 & 4.594 & 1.271 & 133 & 92DA & 6. & 0. \\
\hline $34 *$ & OFB 4 & 14 & 0 . & 34 & 12 & 4.594 & 1.2 & * & 46 & & \\
\hline $35 *$ & 02 & & & 5* & 31 & & & * & $B$ & & \\
\hline 16 & F113 & 2.656 & 0.956 & 86 & F942 & 4.688 & 1.285 & & & & 0.827 \\
\hline $37 \star$ & $113 \mathrm{~F}$ & 2.750 & 1.199 & 87 & D24B & 4.719 & 1.352 & 137* & SAD2 & 6.656 & 0.643 \\
\hline $38 *$ & 80EF & 2.750 & 1.031 & 88 & ODDA & 4.875 & 1.053 & 138* & A6A5 & 6.656 & 0.734 \\
\hline $39 \star$ & CF 30 & 2.781 & 1.111 & 89 & AASA & 4.875 & 1.516 & 139* & B496 & 6.688 & 0.583 \\
\hline 40 & 7730 & 2.781 & 1.111 & 90 & $589 \mathrm{E}$ & 4.875 & 1.083 & 140 & B2CA & 6.688 & 0.527 \\
\hline 41 & EECO & 2.844 & 0.90 & $91 *$ & C237 & 4.938 & 1.144 & & & & \\
\hline 42 & 7466 & 2.844 & 1.093 & 92 & 871 & 4.938 & 1.3 & & & & \\
\hline $43 *$ & $55 \mathrm{AA}$ & 2.875 & 1.34 & 93 & 753 & 5.000 & .2 & & & & \\
\hline 44* & A55 & 875 & 192 & 4* & 359 & 5.063 & 1.36 & & & & \\
\hline $45 *$ & $3 A 8$ & & 1.218 & 95* & A & 063 & 345 & & & & \\
\hline $46 \star$ & $3 \mathrm{C} 3 \mathrm{C}$ & 2.938 & 1.273 & 96 & 9A4B & 5.188 & 1.356 & & & & \\
\hline 47 & F988 & 2.938 & 1.248 & $97 \star$ & F834 & 5.281 & 1.068 & & & & \\
\hline $48 *$ & $6 \mathrm{~F} 60$ & 2.969 & 1.185 & 98 & $9 A 8 D$ & 5.344 & 1.135 & & & & \\
\hline 49 & F660 & 3.000 & 1.090 & 99 & 05B 7 & 5.375 & 0.992 & & & & \\
\hline 50 & E IIE & 000 & 1.031 & $100 *$ & 42E7 & 5.469 & 1.000 & & & & \\
\hline
\end{tabular}

Note-The patterns with an asterisk were used in Part 3.

decimal number, "34B9," where " $B$ " indicates "1011" in binary notation or " 11 "' in decimal notation. The patterns are sorted in ascending order of mean complexity.

Table 3 presents the physical variables employed for analysis. Most of these variables have been regarded in other researches as important in the prediction of pattern goodness or complexity. The variables are defined in the Appendix.

First, a multiple regression analysis was carried out to determine which variables were useful for predicting mean complexity for each pattern. The models that gave high multiple-correlation coefficients were selected from all combinations of the physical variables. The results are shown in Table 4. MSYM2 has the highest correlation coefficient with complexity $\left(\mathrm{R}^{2}=.668, \mathrm{R}=.817\right)$. However, the best two-variable model consists of NTURNS and SUBSYM. The multiple regression equation is

\section{COMPLEXITY}

$$
=0.181 \text { NTURNS }-0.00785 \text { SUBSYM }+2.96 \text {. }
$$

When each variable is standardized so that the mean is 0 and the variance is 1 , the regression equation is 
Table 3

Physical Variables Used for Analysis

\begin{tabular}{|c|c|c|}
\hline Variable Name & Abbreviation & Source \\
\hline Equivalence set size & ESS & Garner \& Clement (1963) \\
\hline Concentration along row or column direction & CRC & Mayekawa (1975) \\
\hline Number of turns & NTURNS & Chipman (1977) \\
\hline Horizontal-vertical symmetry (Type 1) & HVSYM1 & Chipman (1977) \\
\hline Horizontal-vertical symmetry (Type 2 ) & HVSYM2 & \\
\hline Symmetropy (whole) & SYMTRP & Yodogawa (1982) \\
\hline Mean of symmetropy ( $4 \times 4$ windows) & MSYM4 & Yodogawa (1982) \\
\hline Mean of symmetropy $(2 \times 2$ windows $)$ & MSYM2 & Yodogawa (1982) \\
\hline SD of symmetropy ( $4 \times 4$ windows) & SDSYM4 & Yodogawa (1982) \\
\hline SD of symmetropy ( $2 \times 2$ windows) & SDSYM2 & Yodogawa (1982) \\
\hline Saturation of symmetry & SATSYM & \\
\hline Subsymmetry & SUBSYM & \\
\hline
\end{tabular}

\section{COMPLEXITY*}

$$
=0.709 \text { NTURNS* }-0.490 \text { SUBSYM*, }
$$

where an asterisk indicates that the variable is standardized. This model gives a very high multiple-correlation coefficient $\left(R^{2}=.862, R=.928\right)$, and the increase in the multiple-correlation coefficient is very small when one more physical variable is added into the model $\left(\mathbf{R}^{2}\right.$ $=.884, \mathrm{R}=.940$ ).

To understand these results, a factor analysis was conducted. Figure 2 shows factor loadings of the 12 physical variables. In this factor analysis, principal components were extracted and were rotated by the Varimax method. This figure shows that the two-factor solution could be easily interpreted. (The contribution of these two factors reached $71.6 \%$.) NTURNS and CRC load on one factor, which may be concerned with compactness, concentration, or the number of clusters of dots. The variables relating to the degree of symmetry load on the other factor. These two factors will be referred to as "quantitative factor" and "structural factor," respectively. The variables that load on the quantitative factor will be referred to as "quantitative variables," and the variables that load on the structural factor will be referred to as "structural variables."

Our results strongly confirm Chipman's (1977) proposition that physical variables should be classified into quantitative and structural ones. However, it should be pointed out that HVSYM1 and HVSYM2 load on the quantitative factor rather than the structural factor, even though they were originally devised to measure the degree of symmetry. This suggests that searching a pattern with $\mathrm{i} \times 1$ or $1 \times \mathrm{i}$ rectangles $(2 \leq \mathrm{i} \leq \mathrm{n}$, where $\mathrm{n}$ is the number of rows or columns) is not an appropriate method for evaluating the degree of partial symmetry of a twodimensional pattern.

Moreover, mean complexity was added as a variable in factor analysis. As shown in Figure 3, the factor pattern is almost invariant. Note that mean complexity loads on both factors. This figure helps us to understand the results of the multiple regression analysis. The simple correlation of the complexity with MSYM2 or MSYM4 is high, but a linear combination of a quantitative variable and a structural variable leads to a better prediction of complexity. The models containing three or more physical variables cannot give much better predictions, because they share common variances.

\section{Individual Differences}

In order to reveal the source of individual differences, a factor analysis was carried out in which the subjects were regarded as variables and stimulus patterns as cases. The

Table 4

Results of Regression Analysis of All Combinations

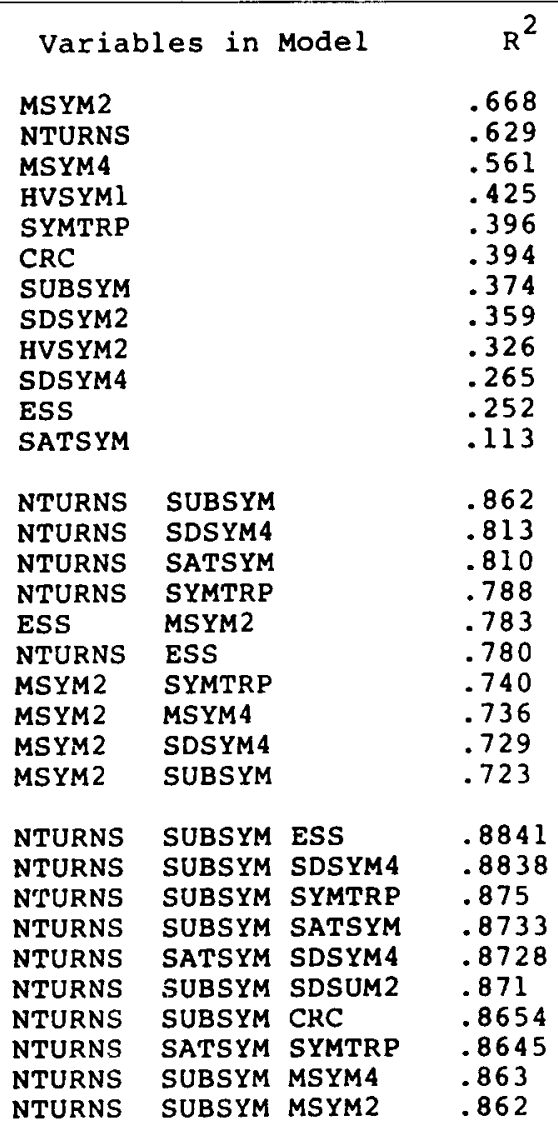




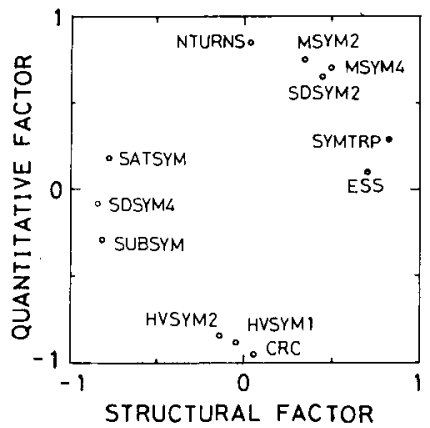

Figure 2. Factor loadings of the 12 physical variables.

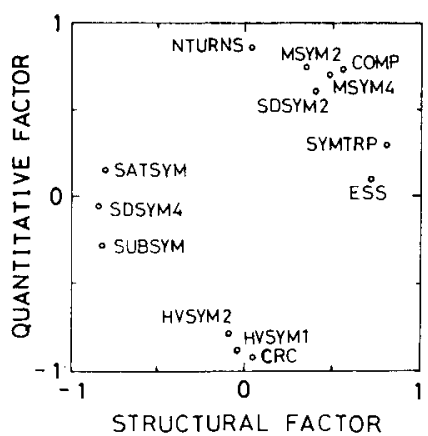

Figure 3. Factor loadings of the 12 physical variables and the mean complexity.

data matrix ( 140 patterns $\times 32$ subjects $)$ was standardized (mean $=0, \mathrm{SD}=1$ ) and the principal component solution was obtained first. Two principal components, which accounted for $78.8 \%$ of the variance, were extracted and rotated by the Varimax method. Figure 4 shows factor loadings of the variables that represent the 32 subjects, and Figure 5 gives factor scores of the 140 stimulus patterns. Each factor can be easily interpreted by viewing Figure 5. The vertical axis seems to represent the number of clusters or concentration of dots in a stimulus pattern, while the horizontal axis seems to represent the degree of structure or organization. So the two factors are called "quantitative factor (psychological)" and "structural factor (psychological)." "Psychological" is added to differentiate these from the factors obtained by the factor analysis of the physical variables. The results of this factor analysis strongly suggest that the individual differences in complexity ratings can be accounted for by the differences in weights on quantitative and structural aspects in a pattern.

How are these psychological factors related to the factors of the physical variables? Another factor analysis was conducted, using the 2 psychological factors together with the 12 physical variables. Figure 6 presents the loadings in the factor analysis, in which principal component analysis and Varimax rotation were employed again. The factor pattern is almost the same as that shown in Figure 2, and quantitative factor (psychological) and structural fac- tor (psychological) load the quantitative factor and the structural factor, respectively, in this factor space. It can be said that the quantitative and structural aspects underlying individual differences nearly correspond to quantitative and structural characteristics measured by the physical variables.

\section{PART 3: EFFECTS OF STIMULUS DURATION}

The analyses in Part 2 suggest that complexity judgment is based on both the quantitative and the structural factors and that the differences in weights on the two factors can account for individual differences in ratings. Moreover, I formulated a working hypothesis that these two factors are derived from two kinds of information processing. Estimation of quantitative features in a stimulus pattern may be caused by primary processing; estimation of structural aspects may be due to higher cognitive processing that involves the discovery of symmetry, repetition, and so on. If stimulus duration is brief, the latter processing will be interrupted, whereas the former processing has already finished; hence subjects cannot find

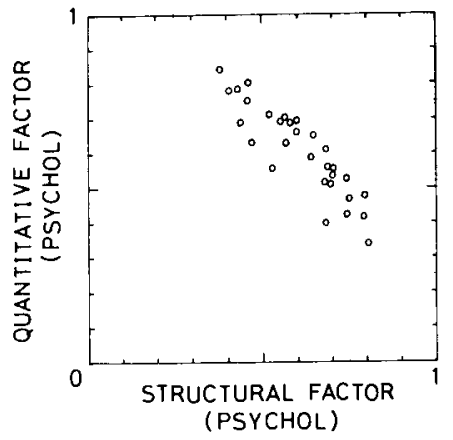

Figure 4. Factor loadings of the 32 subjects.

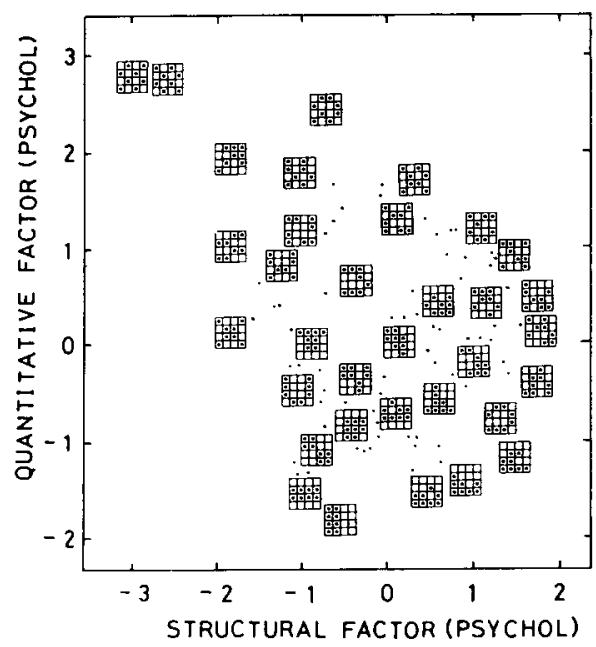

Figure 5. Factor scores of the 140 stimulus patterns. 


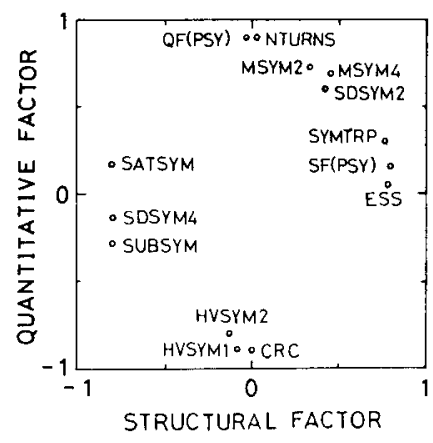

Figure 6. Factor loadings of the 12 physical variables and the two psychological factor scores.

the structure in a stimulus pattern. So it may be expected that the longer the stimulus is presented, the larger will be the effects of structural variables upon complexity ratings. This prediction was tested here.

\section{Method}

Subjects. The subjects were 40 undergraduate students. They were randomly separated into five groups consisting of 8 subjects each.

Materials. From the 140 stimulus patterns used in Part 2, 60 patterns were randomly selected. The patterns employed here are indicated by asterisks in Table 2 . A 35-mm slide was made for each stimulus pattern, with a white background and a black figure. When projected on a screen, the size of each matrix was $16 \times 16 \mathrm{~cm}$ and the diameter of a dot was $2 \mathrm{~cm}$.

Procedure. The stimulus patterns were projected on a translucent screen by a slide projector (Kodak Carousel 2000) with an electric shutter (Takei 270b). The room was always illuminated. The order of the 60 slides was randomly determined and fixed. The subjects were asked to write down a complexity rating for each pattern, using a 7-point scale, immediately after the pattern disappeared. The stimulus duration was $50 \mathrm{msec}, 200 \mathrm{msec}, 1 \mathrm{sec}$, or $4 \mathrm{sec}$, and the interstimulus interval (ISI) was always $4 \mathrm{sec}$. Four groups of subjects were assigned to the four stimulus-duration conditions. The 8 subjects in the other group performed under the condition of 50-msec duration and 8-sec ISI; this condition was designed to test the hypothesis that ISI has no (or small) effects. The experiment was carried out in groups of one through five subjects.

\section{Results and Discussion}

The correlation coefficients of the mean complexity with the physical variables were calculated. In addition, the correlations of the mean complexity with quantitative factor (psychological) and structural factor (psychological) were computed. Partial results are presented in Figure 7, which shows that the mean complexity ratings are highly correlated with the quantitative variables under all conditions of stimulus duration, or that the decrease in coefficients is a possible alternative interpretation of the results shown in Figure 7. On the other hand, the correlation coefficients of the mean complexity with the structural variables increase with stimulus duration. ${ }^{2}$ These results are in accordance with the prediction that the subjects under the condition of short stimulus duration would fail to detect the structure in a stimulus pattern, and would judge complexity mainly by its quantitative features.
The effects of stimulus duration become clearer with another index, "complexity difference," which is defined as the complexity under a limited-duration condition in Part 3 minus the complexity obtained in free rating in Part 2. Figure 8 presents the correlation coefficients of the complexity difference with some variables. For the structural variables, the effects of stimulus duration upon the correlation coefficients are systematic: The absolute values of coefficients decrease with stimulus duration. ${ }^{3}$ This means that the structured patterns were rated to be more complex in short stimulus duration than in free rating. Thus, it is suggested again that the subjects under short-duration conditions cannot sufficiently detect and evaluate the structural aspects in a pattern.

The results for the other condition (50-msec duration and 8-sec ISI) were as follows: The correlation coefficients of complexity were $.798, .728$, and -.641 , with NTURNS, quantitative factor (psychological), and CRC, respectively, and were $.407, .320,-.185$, and -.401 with structural factor (psychological), ESS, SATSYM, and SUBSYM, respectively. So it seems that the differences found among the four groups $(50-\mathrm{msec}, 200-\mathrm{msec}$, 1 -sec, and 4-sec duration) can be ascribed mainly to the stimulus duration itself and not to the intertrial interval. However, note that the results of the 50 -msec-duration,

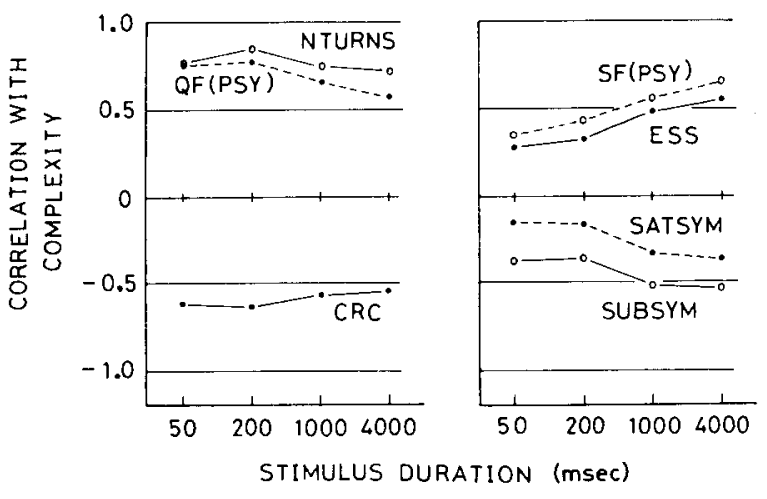

Figure 7. The effects of stimulus duration upon correlation coefincients of complexity with some variables.

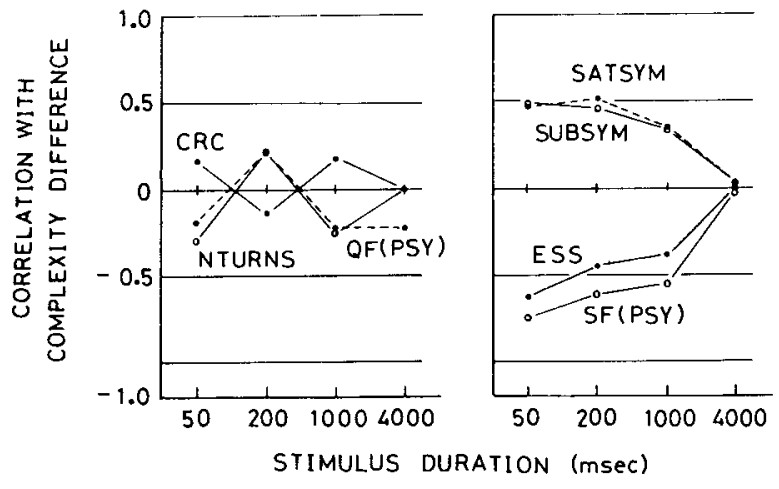

Figure 8. The effects of stimulus duration upon correlation coefficients of "complexity difference" with some variables. 
8-sec-ISI condition were closer to those of the 200-msecduration, 4-sec-ISI condition than to those of the 50-msecduration, 4-sec-ISI condition. This suggests the possibility that subjects can detect some structures from memory images when they have enough time after the offset of stimulus patterns.

\section{GENERAL DISCUSSION}

\section{Summary of the Present Study}

In the present study a framework of quantitative and structural factors in the judgment of pattern complexity, originally advocated by Chipman (1977), was confirmed and further developed. A factor analysis of the 12 physical variables used in pattern psychophysics revealed the quantitative and structural factors. The physical variables could be classified into quantitative and structural variables according to the factor loadings. When the mean complexity was added as a variable in the factor analysis, it loaded on both factors. Multiple regression analysis also showed that the mean complexity was well estimated by a linear combination of a quantitative variable (e.g., NTURNS) and a structural variable (e.g., SUBSYM).

The factor analysis, in which the individual subjects were treated as variables, revealed two factors. The factor scores of the stimulus patterns suggested that the two factors corresponded to the quantitative and the structural factors, respectively. This was confirmed by the factor analysis of these factor scores together with the 12 physical variables. Therefore, the individual differences seem to result from the weights on quantitative and on structural aspects of patterns.

These findings yielded a hypothesis that the quantitative and structural factors are derived from two kinds of information processing: primary processing, which is concerned with the estimation of quantitative features, and higher cognitive processing, which deals with the discovery of structure. So it was expected that the subjects under conditions of short stimulus duration would fail to detect the structure in a pattern and would judge complexity mainly by quantitative features. This prediction was supported by an experiment with limited stimulus $d v$. ration, in which the judged complexity was highly correlated with the quantitative variables under all conditions of stimulus duration, whereas the correlation coefficients of the judged complexity with the structural variables increased with stimulus duration.

\section{A Tentative Model}

Figure 9 shows a model based on the above-mentioned ideas. Two parallel processes are involved in the judgment of pattern complexity. One process detects quantitative features in a pattern (compactness, concentration, the number of clusters of dots, and so on). $Q$ is the output of this process, that is, the estimate of the quantitative aspects of the pattern. The other process detects structural features (symmetry, repetition, and so on). $S$ is the output of this process. The latter process is assumed to require more time than the former. The complexity of a pattern as perceived by a subject is given by $W_{q} Q-W_{s} S$, where $W_{q}$ and $W_{s}$ are the evaluation weights on $Q$ and $S$, respectively. As in Chipman's (1977) hypothesis, it is assumed that the upper limit of perceived complexity is determined by $Q$, and that the discovery of structures reduces perceived complexity. The sources of individual differences exist in the detection of structural features (S) and in the relative weighting of $W_{q}$ and $W_{s}$.

\section{The Process of Detecting Structure}

Most studies in pattern psychophysics have tried to estimate psychological variables using statistical models that contain physical variables as predictor variables. Recent interest has been focused on the information processing used in the perception of complexity, goodness, or similarity (Garner, 1974; Howe, 1980; Howe \& Brandau, 1983; Imai, T. Ito, \& S. Ito, 1976; Klein, 1982). A promising approach is the investigation of differences between individuals, conditions, or developmental stages. The present study suggests that the differences between subjects or between conditions are useful in understanding the information processing used in the judgment of complexity.

On the other hand, Chipman and Mendelson (1979) conducted a developmental study. They examined the in-

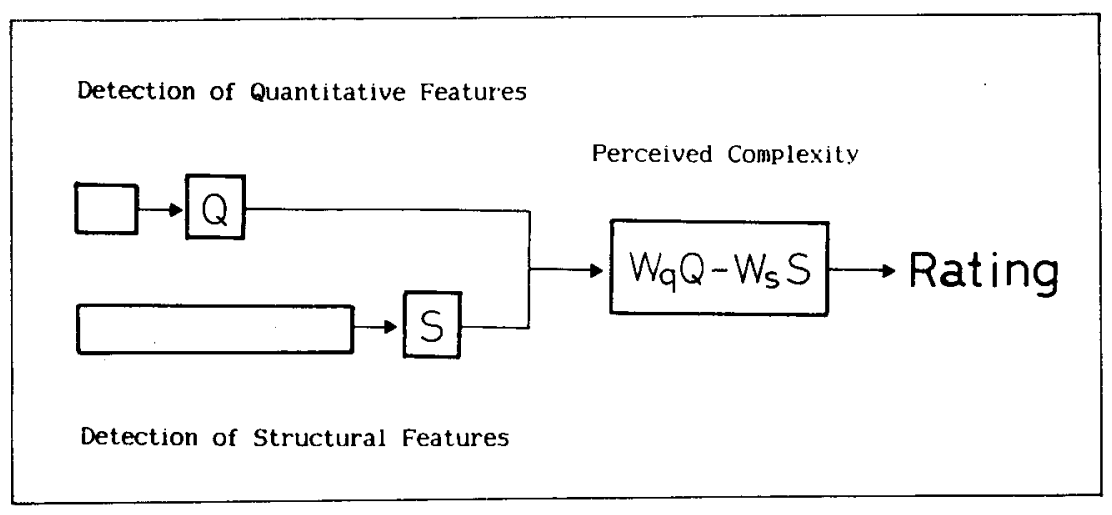

Figure 9. A tentative model for the judgment of pattern complexity. 
fluence of amount of contour and six types of structure of matrix patterns on judged complexity, employing subjects at five grade levels (kindergarten, Grade 2, Grade 4, Grade 6, and college). The effect of amount of contour (perimeter ${ }^{2} /$ area) on judged complexity was highly significant for all age groups. On the other hand, the regression weights for dichotomous variables representing the presence or absence of each type of structure increased with age. This finding is in accordance with the results of the present study. The estimation of quantitative aspects of a pattern is easy and fast in adults, and this type of processing may be formed in an earlier stage of development. The detection of structure in a visual pattern is higher cognitive processing, and it seems to be developed with age.

The process of detecting structure should be considered in detail. Chipman and Mendelson (1979) also showed that only amount of contour, double symmetry, and vertical symmetry significantly affected the judged complexity in the kindergarten group, whereas the effects of the other types of structure (horizontal symmetry, diagonal symmetry, checkerboard organization, and rotational organization) were significant in the older groups. Apparently, not all kinds of structure are equally salient. In fact, many researchers have shown that the time to detect structure in a pattern depends on the type of structure or the orientation of the pattern (Corballis \& Roldan, 1974, 1975; Royer, 1981). The subprocesses, which deal with different kinds of structure, should be considered in a more refined model for complexity judgment.

\section{REFERENCES}

Alexander, C., \& Carey, S. (1968). Subsymmetries. Perception \& Psychophysics, 4, 73-77.

AtTNEave, F. (1957). Physical determinants of the judged complexity of shapes. Journal of Experimental Psychology, 53, 221-227.

Attneave, F., \& Arnoult, M. D. (1956). The quantitative study of shape and pattern perception. Psychological Bulletin, 53, 452-471.

Chipman, S. F. (1977). Complexity and structure in visual patterns. Journal of Experimental Psychology: General, 106, 269-301.

Chipman, S. F., \& Mendelson, M. J. (1979). Influence of six types of visual structures on complexity judgment in children and adults. Journal of Experimental Psychology: Human Perception and Performance, 5, 365-378.

Corballis, M. C., \& Roldan, C. E. (1974). On the perception of symmetrical and repeated patterns. Perception \& Psychophysics, 16, 136-142.

Corballis, M. C., \& Roldan, C. E. (1975). Detection of symmetry as a function of angular orientation. Journal of Experimental Psychology: Human Perception \& Performance, 1, 221-230.

GARNER, W. R. (1974). The processing of information and structure. New York: Halsted Press.

Garner, W. R., \& Clement, D. E. (1963). Goodness of pattern and pattern uncertainty. Journal of Verbal Learning \& Verbal Behavior, 2, 446-452.

HowE, E. S. (1980). Effects of partial symmetry, exposure time, and backward masking on judged goodness and reproduction of visual patterns. Quarterly Journal of Experimental Psychology, 32, 27-55.

HowE, E. S., \& BRANDAU, C. J. (1983). The temporal course of visual pattern encoding: Effects of pattern goodness. Quarterly Journal of Experimental Psychology, 35A, 607-633.

ICHIKAWA, S. (1981). Rated ease of memorization of dot-in-matrix patterns: Multiple regression analysis by physical variables which describe configuration of dots. Japanese Psychological Research, 23, 69-78.
ICHIKAWA, S. (1982). Measurement of visual memory span by means of the recall of dot-in-matrix patterns. Behavior Research Methods \& Instrumentation, 14, 309-313.

ICHIKAWA, S., \& GYOBA, J. (1984). Methodological problems on pattern psychophysics. Japanese Psychological Review, 27, 132-157.

ImAI, S., Iтo, T., \& Iтo, S. (1976). Effect of intra-pattern transformation structures upon goodness judgments of two-dimensional patterns. Japanese Journal of Psychology, 47, 202-210.

KLEIN, R. (1982). Patterns of perceived similarity cannot be generalized from long to short exposure durations and vice versa. Perception \& Psychophysics, 32, 15-18.

MayeKaWA, S. (1975). Physical and psychological structures of dot patterns. Unpublished bachelor's thesis, University of Tokyo.

ROYER, F. L. (1981). Detection of symmetry. Journal of Experimental Psychology: Human Perception and Performance, 7, 1186-1210.

YodoGawa, E. (1982). Symmetropy, an entropy-like measure of visual symmetry. Perception \& Psychophysics, 32, 230-240.

\section{NOTES}

1. The Spearman-Brown reliability coefficient of the mean complexity was .985 when the subjects were randomly split into two groups. The correlation coefficient between the complexity grade in Part 1 and the mean complexity was .796 .

2. The differences in correlation coefficients between the 50 -msec condition and the 4-sec condition were tested. The degree of freedom is 57. For NTURNS and CRC, the differences were not significant $(t=1.46$ and $t=1.54$, respectively). SUBSYM reached a $5 \%$ level of significance ( $t=2.24$, two-tailed), and the differences were highly significant for the other variables [ $t=3.52, \mathrm{p}<.001$ for $\mathrm{QS}(\mathrm{PSY})$; $\mathrm{t}=6.64, \mathrm{p}<.001$ for $\mathrm{SF}(\mathrm{PSY}) ; \mathrm{t}=4.40, \mathrm{p}<.001$ for ESS $\mathrm{t}=$ $3.09, \mathrm{p}<.005$ for SATSYM].

3. The differences in the correlation coefficients between the $50-\mathrm{msec}$ condition and the 4-sec condition were not significant for NTURNS, $\mathrm{QS}(\mathrm{PSY})$, and CRC $(\mathrm{t}=1.67, \mathrm{t}=.26, \mathrm{t}=.92$, respectively). For the structural variables, the differences were highly significant $[t=7.33$, $\mathrm{p}<.001$ for $\mathrm{SF}(\mathrm{PSY}) ; \mathrm{t}=4.82, \mathrm{p}<.001$ for ESS; $\mathrm{t}=2.78, \mathrm{p}<$ .01 for SATSYM; $\mathrm{t}=2.93, \mathrm{p}<.005$ for SUBSYM].

\section{APPENDIX}

\section{Definitions of the Physical Variables}

The definitions of the physical variables used to describe dotin-matrix patterns are briefly stated. It is supposed that the original pattern consists of $\mathrm{d}$ dots in an $\mathrm{n} \times \mathrm{n}$ matrix.

ESS (equivalence set size). ESS is the number of patterns produced by transformations using reflection and $90^{\circ}$ step rotation on a given pattern. The values of ESS are 1, 2, 4, or 8 for 8-dot-in- $4 \times 4$-matrix patterns, and a small ESS indicates that the pattern is more symmetrical. See Garner and Clement (1963) or Royer (1981) for details.

CRC (concentration along row or column direction). CRC is a function of CR and CC. CR represents the degree of concentration of dots along row direction. Let $d_{i}$ be the number of dots contained in row $i(1 \leq i \leq n)$, and let $p_{i}=d_{i} / d$. Then,

$$
\mathrm{CR}=1-\left[\sum_{\mathrm{i}=1}^{\mathrm{m}} \mathrm{p}_{\mathrm{i}} \log _{2}\left(1 / \mathrm{p}_{\mathrm{i}}\right)\right] / \log _{2} \mathrm{~m} .
$$

If $p_{i}=0, p_{i} \log _{2}\left(1 / p_{i}\right)=0 . A$ large $C R$ indicates that dots are more concentrated in particular rows. $\mathrm{CC}$ represents the degree of concentration of dots along column direction. Then,

$$
\mathrm{CRC}=1-(1-\mathrm{CR})(1-\mathrm{CC}) .
$$


Accordingly, CRC increases with CR and CC, and it becomes 1 if either CR or CC is 1 . See Ichikawa $(1981,1982)$ for details.

NTURNS (number of turns). When two adjacent sides of a cell are on the boundary between filled and empty areas, one turn is counted. This measure was originally used not for dot patterns but for block patterns by Chipman (1977).

HVSYM1 (horizontal-vertical symmetry, type 1). This measure was developed by Chipman (1977) to detect partial symmetry as well as overall symmetry. A given pattern is scanned through all possible $i \times 1$ or $1 \times i$ rectangles $(2 \leq i \leq n)$, and the number of symmetric rectangles is counted. To calculate HVSYMI, each symmetry is weighted by the area of the rectangle. This measure is an application of Alexander and Carey's (1968) "subsymmetry," which was devised for one-dimensional patterns.

HVSYM2 (horizontal-vertical symmetry, type 2). Each symmetry is weighted by the square of the area of the rectangle. HVSYM2 is calculated so that the symmetry of a large rectangle has more weight.

SYMTRP (symmetropy of the whole pattern). The symmetropy was developed by Yodogawa (1982) to measure the amount of symmetry using the two-dimensional Walsh transform and the entropy in information theory. The two-dimensional discrete Walsh function $W_{m, n}(i, j)$ of order $(m, n)$ and argument $(i, j)$ is defined as

$$
\begin{gathered}
w_{m, n}(i, j)=w_{n}(i) w_{m}(j), \\
m, n, i, j,=0,1,2, \ldots, N-1,
\end{gathered}
$$

where $N=2^{q}$ ( $q$ is a positive integer), and $w_{n}(i)$ and $w_{m}(j)$ are the one-dimensional discrete Walsh functions. The two-dimensional Walsh functions are vertically symmetric if $m$ is even and $\mathrm{n}$ is odd, horizontally symmetric if $\mathrm{m}$ is odd and $\mathrm{n}$ is even, centrosymmetric if both $\mathrm{m}$ and $\mathrm{n}$ are odd, or doubly symmetric if both $\mathrm{m}$ and $\mathrm{n}$ are even. The component of each type of symmetry $\left(\mathrm{P}_{\mathrm{k}}, \mathrm{k}=1,2,3,4\right)$ can be obtained based on the Walsh power spectra. Then the symmetropy is defined as

$$
\text { SYMTRP }=\sum_{k=1}^{4} P_{k} \log _{2}\left(1 / P_{k}\right)
$$

MSYM4 (mean of symmetropy observed through $4 \times 4$ windows) and MSYM2 (mean of symmetropy observed through $2 \times 2$ windows). Yodogawa (1982) introduced the observation windows to measure partial symmetry. They are the $2^{\mathrm{k}} \times 2^{\mathrm{k}}(\mathrm{k}=$ $1,2, \ldots)$ square regions through which a pattern is observed at all possible distinct window positions. In the present study, the original $4 \times 4$ pattern was embedded into a $6 \times 6$ pattern, and the mean symmetropy value (averaged over all possible window positions) was computed.

SDSYM4 (standard deviation of symmetropy observed through $4 \times 4$ windows) and SDSYM2 (standard deviation of symmetropy observed through $2 \times 2$ windows). The standard deviations were also introduced by Yodogawa (1982) in order to estimate the homogeneity of a pattern for each window size.

SATSYM (saturation of symmetry). The saturation of a given kind of symmetry is the proportion of the area of the largest symmetric rectangle (or square) in a pattern. For example, if the largest and vertically symmetric rectangle in a $4 \times 4$ pattern is $3 \times 4$, then the saturation of vertical symmetry is $12 / 16=.75$. SATSYM is the sum of the saturation of vertical, horizontal, upper-right diagonal, upper-left diagonal, and $180^{\circ}$ rotational symmetry.

SUBSYM (subsymmetry). This measure is also an application of Alexander and Carey's (1968) subsymmetry. A given pattern is scanned by $\mathrm{i} \times \mathrm{j}$ rectangles $(2 \leq \mathrm{i} \leq \mathrm{n}, 2 \leq \mathrm{j} \leq \mathrm{n})$. If a rectangle is symmetric, a score $i \times j$ is given. The kinds of symmetry are same as those of SATSYM if $i=j$, and only vertical, horizontal, and $180^{\circ}$ symmetry are considered if $\mathrm{i} \neq \mathrm{j}$. Each symmetry is scored independently, so double symmetry gives more scores.

(Manuscript received June 7, 1985; revision accepted for publication August 9, 1985.) 